\title{
S-A-5. A Study of Subacute Intracranial Hematoma after Head Trauma
}

\author{
Shinichiro ENDO \\ Surgical Department, Fukusihma Medical School
}

Selected cases of head injuries with intracranial hematoma treated operatively at subacute stage following trauma were studied. These cases had several characteristic points as follows:

1) The cases with extradural hematomas were more common than the one with subdural hematomas.

2) Pathological findings of pupils were observed in a few cases at the late stage after trauma, but in almost all cases no changes of pupils were induced.

3) Typical contralateral motor weakness was not found in almost all cases.

4) The cerebrospinal fluid was hypertensive in one half of the cases and normotensive or hypotensive in another half, and had bloody components in one half and none bloody in another half.

5) Abnormal findings of cerebral angiograms were demonstrated in almost all cases. However, the abnormal changes of many cases were so slight that these were apt to be overlooked.

6) Skull fractures were observed in almost all cases. These fractures were divided into two groups according to the locations of them; the one group was lineal fracture or depressed fracture at the frontal or occipital part and the another group was lineal fracture pararell to the direction of middle meningeal artery. Accordingly fractures in both groups were not crossed with the main branch of middle meningeal artery.

\section{S-A-6. Importance of Cerebral Carotid Angiography in Severe Head Injury, as an Indication of Surgical Intervention}

\author{
Seiya Shirakata, Shigekiyo Fujita, Kiyoto Takao, Seiji Shinmaru, \\ Hideji Hiromoto, Kazuhiko Hitomi and Takao Mrtsuno \\ 1st Department of Surgery, Kobe University School of Medicine
}

Recently, severe head injuries accompanied with intracranial hematoma and cerebral contusion has increased tremendously in comparision to the typical hematoma. It is of great importnce to determine the indication of operation for 
treatment of these patients. Cerebral carotid angiography is one of the most practical and useful method of diagnosis for these cases.

The findings of cerebral carotid angiography were studied from the operative findings or through autopsies and compared with the clinical symptoms in dividing into the living and dead group.

The results of 42 cases (cerebral contusion 9, intracranial hematoma 33) were as follows:

1) In frontal view, the shift of anterior cerebral artery $\left(A_{1}-A_{5}\right)$ were recognized in 10 out of 14 cases in the living group, but all 19 cases in the dead group. Angle formation at the transition of $A_{1}$ and $A_{2}, A_{2}$ and $A_{3}$ which is called Cognack Schwenker's Bild by Huber was seen in 2 cases of the living group, but 6 cases in the dead group. Arch formation was seen only in the 6 dead cases. Angle formation is said to be characteristic in extradural hematoma and intracerebral hematoma of frontal region, but these changes in the anterior cerebral artery are considered to be emphasized by cerebral contusion and the resulting cerebral edema.

2) In frontal view, the shift of the terminal internal carotid artery toward the mid-line is usually recognized in hematomas of the temporal region, but these findings have also been in hematomas of other region in the dead group. 12 cases of the 19 dead cases presented this change and especially in 4 cases, the shift approached to the mid-line. These changes are considered to be caused by cerebral contusion and the resulting cerebral edema.

3) In lateral view, the sylvian group of middle cerebral artery had a tendency to be straight in the dead group.

4) Visualization of the posterior cerebral artery were gained in $50 \%$. Findings of transtentorial herniation were seen in 6 out of the 7 dead cases.

It may be summarized that cerebral carotid angiography is of great importance and useful not only to seek the location of hematomas but also to diagnose the indication of operation, to predict he prognosis.

\title{
S-A-7. Quick Diagnosis of Intracranial Hematomas by Ultrasound
}

\author{
Kazufumi Ito, Yuichi AbE and Kenji TanaKa
}

Neurosurgical Clinic, Department of Surgery, Juntendo University School of Medicine

The clinical values of he ultrasonic diagnosis of intracranial hematoma by means of pulse method will be presented.

The transducer was used barium titanate crystal of $10 \mathrm{~mm}$ in diameter at a 